\title{
Fabrication and coupling to planar high- $Q$ silica disk microcavities
}

\author{
T. J. Kippenberg, S. M. Spillane, D. K. Armani, and K. J. Vahala ${ }^{a)}$ \\ Department of Applied Physics, California Institute of Technology, Pasadena, California 91125
}

(Received 24 March 2003; accepted 20 May 2003)

\begin{abstract}
Using standard lithographic techniques, we demonstrate fabrication of silica disk microcavities, which exhibit whispering-gallery-type modes having quality factors $(Q)$ in excess of 1 million. Efficient coupling (high extinction at critical coupling and low, nonresonant insertion loss) to and from the disk structure is achieved by the use of tapered optical fibers. The observed high $Q$ is attributed to the wedged-shaped edge of the disk microcavity, which is believed to isolate modes from the disk perimeter and thereby reduce scattering loss. The mode spectrum is measured and the influence of planar confinement on the mode structure is investigated. We analyze the use of these resonators for very low loss devices, such as add/drop filters. (C) 2003 American Institute of Physics. [DOI: 10.1063/1.1593833]
\end{abstract}

Microcavities are of interest for a wide range of fundamental and applied studies, including investigations of cavity quantum electrodynamics, ${ }^{1-4}$ optical elements for telecommunications, ${ }^{5}$ single-photon sources, ${ }^{6,7}$ and chemical/biological sensors. ${ }^{8}$ Microfabrication techniques allow reproducible fabrication of resonators with lithographically controlled dimensions, and whispering gallery (WG) devices have the highest quality factor $(Q)$ to date. Using a combination of lithography and etching, semiconductor WG microcavities have obtained $Q$ values in the range of $10^{4}$ (Ref. 9), while polymer WG cavities ${ }^{10}$ have recently obtained a $Q$ value of $10^{5}$. $Q$ values are typically limited by scattering at the all-important resonator perimeter. As a way to overcome this problem, we have recently reported a technique to fabricate ultra-high- $Q$ (exceeding $10^{8}$ ) toroid microcavities on a chip. ${ }^{11}$ In that work, a laser-assisted reflow step is used to achieve the atomically smooth resonator surface finish characteristic of ultra-high- $Q$ surface-tension-induced microcavities. In this letter, we demonstrate thatsurprisingly_planar silica disk resonators can exhibit whispering gallery modes (WGMs) modes with high- $Q$ values in excess of 1 million, without any additional chemical or laser processing. The spectral properties of these resonators are very regular and reproducible, and are readily modeled. Furthermore, the choice of silica as the resonator material has the advantage of a large transparency window (extending from the visible to the near-infrared) as well as a very low intrinsic material loss. Additionally, compatibility with fiberoptic technologies is demonstrated by efficient coupling to these resonators through a tapered optical fiber.

Silica microdisk resonators were fabricated using a combination of optical lithography, wet oxide etching and a dry silicon-etching process. First, silicon wafers with $2 \mu \mathrm{m}$ of thermally grown oxide were patterned with circular photoresist pads by standard lithographic techniques. The samples were then etched with buffered HF until the nonpatterned oxide was completely removed, leaving the lithographically defined circular silica disks. The HF etch leaves the silica disk with wedge-shaped edges, caused by undercutting of the

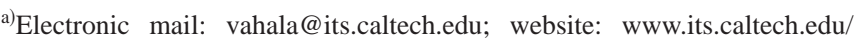
$\sim$ vahalagr
}

photoresist pads. The silica disks were then isolated from the silicon substrate with an isotropic silicon dry etch $\mathrm{XeF}_{2}$, leaving the resonator suspended by a circular pillar (Fig. 1). Disk resonators support WG optical modes that are confined near the disk perimeter. To analyze the $Q$ factor of these modes as well as the mode spectrum, tapered optical fibers ${ }^{12}$ were used to couple to the disk resonator. Briefly, tapered optical fibers are made by heating and stretching a standard SMF-28 telecommunication fiber, while monitoring the optical transmission. By keeping an adiabatic taper profile, the fundamental core-guided mode of a standard SMF fiber can be converted to the air-guided fundamental taper mode $H E_{11}$, and vice versa, with negligible loss. The tapered fiber was attached to a piezoelectric stage which allows precise positioning of the taper with respect to a silicon wafer containing multiple microdisks. An optical micrograph of a taper coupled to a disk is shown in the inset of Fig. 1.

When brought in the proximity of the microdisk, the taper mode can evanescently excite a WGM. The efficiency of this process depends on the ratio of parasitic loss (e.g., undesired coupling from either the fundamental taper mode or the target resonator mode to higher-order taper modes or radiation modes) to the intended taper-junction coupling. The efficiency of this junction in the context of coupling to high- $Q$ microresonators has been studied in reference. ${ }^{15}$ Fig-

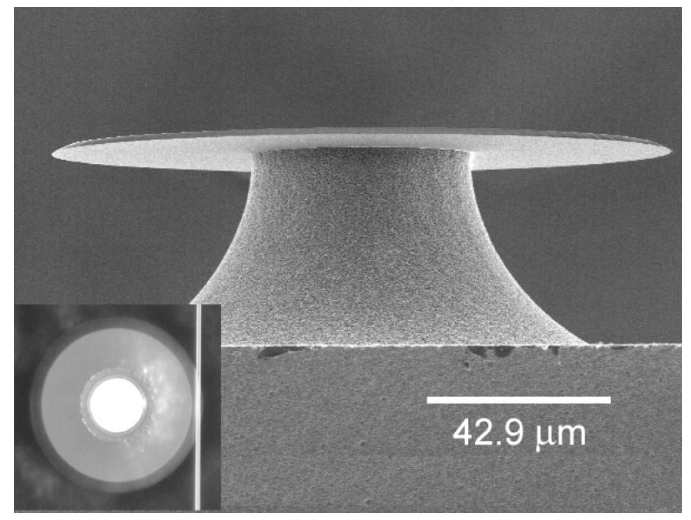

FIG. 1. SEM micrograph of a $\sim 120$ - $\mu \mathrm{m}$-diameter microdisk resonator (2$\mu \mathrm{m}$-thick oxide disk). The inset shows an optical micrograph of a resonator coupled to a tapered optical fiber. 


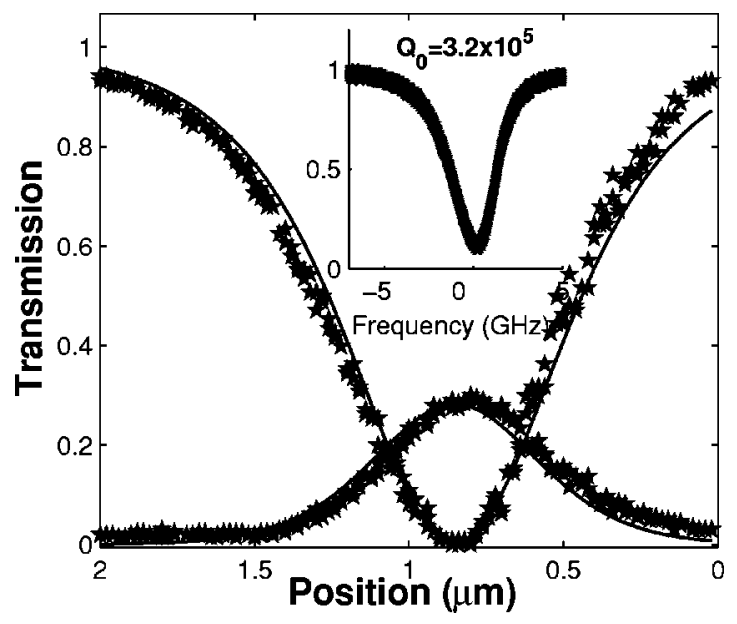

FIG. 2. Transmission (normalized with respect to the launched fiber power) and reflection properties versus taper-disk gap distance. The inset shows the transmission versus frequency near the critical point. The off-resonant loss was less than $5 \%$.

ure 2 shows the absolute transmission and reflection properties at resonance as a function of the taper-disk gap of a microdisk resonator side-coupled via a tapered fiber. As the taper is progressively moved closer to the resonator (step size being $20 \mathrm{~nm}$ ), the transmission transits different coupling regimes, from undercoupled to critically coupled to overcoupled. ${ }^{13}$ Nearly complete extinction is reached at the critical point $(>99 \%)$. When further reducing the gap distance, the overcoupled transmission approaches unity (the actual measured value is $>93 \%$ ). In addition, bringing the taper close to the microdisk caused negligible scattering as observed by the small increase in off-resonant loss of $<5 \%$. The fact that strong overcoupling can be achieved, with negligible off-resonant loss, demonstrates the high efficiency of the tapered-fiber-coupling junction, which is $>93 \%$ in this case. $^{15}$

The $Q$ factor of WG resonances was inferred by linewidth measurements, using a $300-\mathrm{KHz}$ external-cavity diode laser to excite the resonances in the 1550 -nm band. $Q$ factors above 1 million were consistently observed, the highest observed value to date being $3.2 \times 10^{6}$. We attribute the high $Q$ factor, despite the lithographic roughness, to the wedgeshaped feature noted earlier. The wedge is believed to induce modal isolation from the disk edge, where etch blemishes are most pronounced.

Due to a combination of perimeter roughness and intrinsic thermal-oxide inhomogeneities, a fraction of the circulating power is backscattered into the mode that counterpropagates with respect to the mode excited by the taper. This other mode couples out of the resonator in the reverse direction along the fiber, equivalent to a reflection. ${ }^{14}$ The transmission $(T)$ and reflection $(R)$ through the resonatorwaveguide system in the presence of backscattering are given by

$$
\begin{aligned}
& T=\left[\frac{\Gamma^{2}+(1-K)(1+K)}{\Gamma^{2}+(1+K)^{2}}\right]^{2} \\
& R=\left[\frac{2 \Gamma K}{\Gamma^{2}+(1+K)^{2}}\right]^{2} .
\end{aligned}
$$

Here, $\Gamma$ accounts for the excitation of the counterpropagating Downloaded 20 Dec 2005 to 131.215.225.171. Redistribution subje

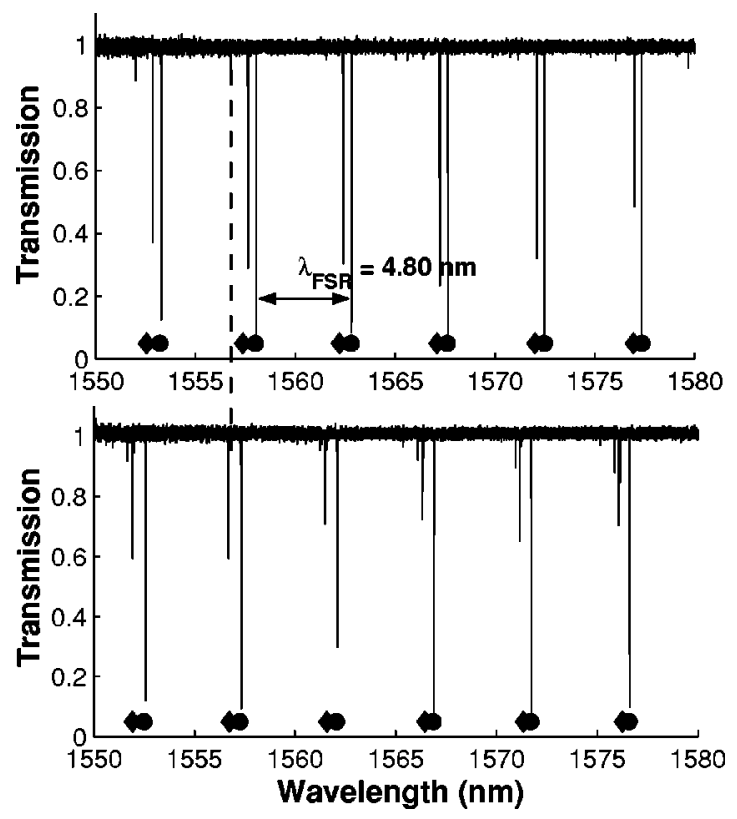

FIG. 3. Mode spectrum of a 114- $\mu$ m-diameter resonator for TE and TM polarizations. The circles and diamonds represent the first- and second-order radial modes, based on modeling.

waveguide mode, and $K$ is the coupling strength, defined as $K \equiv \tau_{0} / \tau_{\text {ex }}$ (i.e., the ratio of intrinsic lifetime to the coupling lifetime). The $K$ parameter typically varies exponentially with waveguide-resonator separation. The $\Gamma$ parameter can be readily measured in the undercoupled regime by observing the associated resonant splitting, ${ }^{14}$ and was approximately 1.5 for the device measured in Fig. 2. The observed reflection of approximately $28 \%$ is consistent with this $\Gamma$ value using theory. We have observed $\Gamma$ parameters up to 3.2 for structures of similar dimensions. The solid lines in Fig. 2 represent a theoretical fit using Eq. (1).

We also investigated the mode structure of the samples. Figure 3 shows the measured cavity mode spectrum for a $114-\mu \mathrm{m}$-diameter resonator (2- $\mu \mathrm{m}$-oxide thickness). The dimensions were measured by scanning-electron-microscope (SEM) studies. The spectrum was taken for both polarizations (TE/TM) supported by the microdisk resonator. The free spectral range (FSR, modes with successive angular mode numbers) was measured to be $\Delta \lambda_{\mathrm{FSR}}=4.80 \mathrm{~nm}$. We inferred the approximate center of the radial field distribution from the FSR $\Delta \lambda_{\mathrm{FSR}} \approx \lambda^{2} / 2 \pi R n$ using the refractive index of thermally grown oxide $n=1.445$. The resulting diameter of $109 \mu \mathrm{m}$ lies between the measured inner wedge edge (100 $\mu \mathrm{m})$ and outer $(114 \mu \mathrm{m})$ diameter of the disk, suggesting that the modes are confined to within inner and outer radii of the wedge. A further indication is that the maximum coupling strength of these modes was achieved with the tapered fiber located tangentially near the center between inner and outer radii. These findings are consistent with the conjecture concerning the origin of the high modal $Q$.

As the WGMs of this structure cannot be solved analytically, approximate solutions were obtained using a simplified disk model. Identifications using this model are given in Fig. 3 with first- and second-order radial modes denoted using circles and diamonds. The TE/TM spectra exhibit slightly different splitting of the radial modes $(0.6$ and $0.8 \mathrm{~nm}$, respectively). In addition, the influence of vertical confinement to AIP license or copyright, see http://apl.aip.org/apl/copyright.jsp 
on mode spectra was studied. In experiments two samples having layer thicknesses of 1 and $2 \mu \mathrm{m}$ were measured. The resulting spectra exhibit qualitatively the same structure as shown in Fig. 3. However, as the oxide thickness is reduced from 2 to $1 \mu \mathrm{m}$, the splitting of the two dominant radial modes increases significantly for one polarization, while remaining practically unchanged for the other.

The high- $Q$ devices demonstrated here can be important for low insertion loss devices such as add/drop filters, which typically require large bandwidth and correspondingly low total $Q$. In a symmetric add/drop device, efficient channel dropping requires that the total $Q$ factor of the resonator is primarily induced by loading. If this is not the case, parasitic cavity loss will induce an excessive loss penalty upon channel drop as well as an extinction-ratio penalty. For example, consider the ratio of power dropped versus incoming waveguide power, in a device consisting of two identical waveguides symmetrically coupled to a resonator. A simple analysis gives

$$
\frac{P_{\text {dropped }}}{P_{\text {in }}}=1-\frac{4 Q_{0} / Q_{\mathrm{ex}}}{\left(1+2 Q_{0} / Q_{\mathrm{ex}}\right)^{2}}-\frac{1}{\left(1+2 Q_{0} / Q_{\mathrm{ex}}\right)^{2}} .
$$

Here, the first term constitutes a loss incurred because the resonator has a finite $Q_{0}$, and the second term is a loss resulting from residual transmission. As is evident from the expression, a high intrinsic-to-external $Q$ ratio will minimize insertion loss of the dropped signal by first reducing the impact of intrinsic resonator loss, and second by biasing the input waveguide towards the optimal critical-coupling point, thereby coupling more power into the resonator. For asymmetrically coupled waveguides, the finite channel transmission can be remedied by critically coupling the input waveguide to the resonator. The insertion loss of the dropped channel in this case is composed only of intrinsic resonator loss, which can be minimized using a large intrinsic $Q$ :

$$
\frac{P_{\text {dropped }}}{P_{\text {in }}}=1-\frac{1}{\left(1+Q_{0} / Q_{\mathrm{ex}}\right)} .
$$

Using the highest $Q$ values observed in this work and assuming a loaded $Q$ of $2 \times 10^{4}$ (corresponding to a dropped bandwidth of $10 \mathrm{GHz}$ ) yields a channel extinction of $50 \mathrm{~dB}$ and a drop loss of $0.03 \mathrm{~dB}$ for a symmetrically configured add/drop filter. For comparison, an intrinsic $Q$ factor of $2 \times 10^{4}$ yields an extinction of $9.5 \mathrm{~dB}$ and a drop loss of $3.5 \mathrm{~dB}$.
In summary, we have fabricated high- $Q$ silica resonators on a chip. A combined wet etch and isotropic gas etch leaves undercut silica disks with a silicon support pillar. Without any further surface processing, quality factors in excess of $10^{6}$ were measured. These high $Q$ values are attributed to mode isolation away from the disk edge that is induced by a wedge-shaped disk profile. High $Q$ values can be used to create very low loss and high extinction add/drop filters, even in devices which operate at a substantially lower loaded $Q$ value. In addition, we have shown that tapered fibers enable efficient excitation of the disk modes as well as strong overcoupling with low, off-resonant insertion loss. Additional optical functionality in these structures can be added by means of implantation to obtain low threshold lasers.

We appreciate and thank William Green and Alireza Ghaffari for their help and advice in microfabrication. This research was funded by the DARPA, the NSF, and CALTECH Lee Center for advanced networking.

${ }^{1}$ Lefevre-Seguin and V. Haroche, Mater. Sci. Eng., B 48, 53 (1997).

${ }^{2}$ W. Hansel, P. Hommelhoff, T. W. Haensch, and J. Reichel, Nature (London) 413, 498 (2001).

${ }^{3}$ D. W. Vernooy, A. Furusawa, N. P. Georgiades, V. S. Ilchenko, and H. J. Kimble, Phys. Rev. A 57, R2293 (1998).

${ }^{4}$ V. Zwiller, S. Falth, J. Persson, W. Seifert, L. Samuelson, and G. Bjork, J. Appl. Phys. 93, 2307 (2003).

${ }^{5}$ R. E. Slusher, A. F. J. Levi, U. Mohideen, S. L. McCall, S. J. Paerton, and R. A. Logan, Appl. Phys. Lett. 63, 1310 (1993).

${ }^{6}$ M. Pelton, C. Santori, G. Solomon, O. Benson, and Y. Yamamoto, Eur. Phys. J. D 18, 179 (2002).

${ }^{7}$ P. Michler, A. Kiraz, C. Becher, L. Zhang, E. Hu, A. Imamoglu, W. Schoenfeld, and P. Petroff, Phys. Status Solidi B 224, 797 (2001).

${ }^{8}$ F. Vollmer, D. Braun, A. Libchaber, M. Khoshsima, I. Teraoka, and S. Arnold, Appl. Phys. Lett. 80, 4057 (2002).

${ }^{9}$ B. Gayral, J. G. A. Lemaitre, C. Dupuis, L. Manin, and J. L. Pelouard, Appl. Phys. Lett. 75, 1908 (1998).

${ }^{10}$ P. Rabiei, W. H. Steier, C. Zhang, and L. R. Dalton, J. Lightwave Technol. 20, 1968 (2000).

${ }^{11}$ D. K. Armani, T. J. Kippenberg, S. M. Spillane, and K. J. Vahala, Nature (London) 422, 925 (2003).

${ }^{12}$ M. Cai, O. Painter, and K. Vahala, Phys. Rev. Lett. 85, 74 (2000).

${ }^{13}$ H. A. Haus, Waves and Fields in Optoelectronics (Prentice-Hall, Englewood Cliffs, NJ, 1984).

${ }^{14}$ T. J. Kippenberg, S. M. Spillane, and K. J. Vahala, Opt. Lett. 27, 1669 (2002).

${ }^{15}$ S. M. Spillane, T. J. Kippenberg, O. J. Painter, and K. J. Vahala, Phys. Rev. Lett. (accepted for publication). 